Objectives The evolution of nerve conduction studies and EMG was determined following 30 patients with chronic renal failure maintained on regular haemodialysis for 12 men for 12 months. Methods Eighteen different electroneurographic (ENG) indices were compared in three groups of patients of different dialysis duration.

Results No relationship was found between neuropathy and duration of haemodialysis. No correlation was found between all ENG indices and the age and sex. Also, nerve conduction was independent of the disease underlying renal failure. The side of arteriovenous vascular access had no statistically significant effect on motor nerve conduction studies in the upper extremities.

ENG indices showed no significant correlation with serum creatinine, PTH, and beta 2 microglobulin.

Mean motor nerve conduction velocity (MNCV) and distal latency (DL) were significantly different (decreased and increased respectively) at the beginning of the study and one year later for the median, ulnar, and common peroneal nerves on both sides. The change was more in patients with low $\mathrm{Kt} / \mathrm{V}$ (a depuration index) than those with high $\mathrm{Kt} / \mathrm{V}$.

Conclusion At the end of the study all patients had had peripheral polyneuropathy, 8 patients $(26.7 \%)$ had got CTS, and 8 patients $(26.7 \%)$ had subclinical median mononeuropathy. Axonal neuropathy was present in $100 \%$ of patients and demyelinating neuropathy was present in 8 patients $(26.7 \%)$.

\section{AB0194 USES OF MAGNETIC RESONANCE IMAGING (MRI) AND MYELOGRAPHY IN IRAQI PATIENTS WITH PROLAPSED INTERVERTEBRAL DISC (PID) AND THE CORRELATION OF JOINT MIBILITY TO PID}

ZS Al-Rawi, SH Al-Amiedy, A Kammona. Department of Medicine and Radiology, College of Medicine, University of Baghdad, Baghdad, Iraq

\subsection{6/annrheumdis-2001.672}

\section{Background}

Objectives To compare between the uses and side effects of MRI and myelography for the diagnosis of PID, and to assess the relation between PID and joint mobility.

Methods One hundred thirty patients with PID proved by MRI (65) on myelography (65) were compared with that in an age and sex matched healthy 130 individuals without PID joint mobility was scored on a scale of $0-8$ using the method described by Reighton et al.

Results PID was commonly reported at L4/5 or L5/S1 levels, whilst other levels were less frequently involved. Both imaging modalities were highly sensitive to prove the diagnosis of PID and its level, unfortunately myelography showed significantly more side effects compared to MRI.

The number of patients with hypermobile joints and the total mobility scores were comparable in patients and control groups. Conclusion This study showed that both imaging modalities are sensitive for diagnosis of PID, and MRI is a safer technique compared to myelography. There is no relation between PID and joint mobility.

\section{AB0195 WHIPLASH FROM HEAD TO TOE}

${ }^{1} \mathrm{~N}$ Yanes-Hoffman, ${ }^{2} \mathrm{DM}$ Fraser. ${ }^{1}$ Orthopaedic Medicine, NYH HealthCare Communications Group, Rochester, USA; ${ }^{2}$ Orthopaedic Medicine, Orthopaedic Medicine Clinic, St Catharines, Ontario, Canada

\subsection{6/annrheumdis-2001.673}

Background Although "Whiplash" is an overworked term, it is a troublesome, underdiagnosed, undertreated problem. Unless cervical "straightening" indicates muscle spasm secondary to softtissue injury, X-rays, CT scans, MRIs contribute little diagnostic information.

To diagnose true ?whiplash,? the old-fashioned fundamentals of medical practice?-a careful history and a thorough physical examination-are necessary.

Objectives Evaluating Cyriax's dicta that: 1) examining the neck alone for post-traumatic, soft-tissue damage is an inadequate diagnostic approach; 2) since "All muscle spasm is secondary," the source of the spasm must be found to diagnose and treat the condition.

Methods From 1-1-97 to 1-1-2000, 197 patients presented with musculoskeletal complaints of the neck secondary to trauma from an automobile accident.

Although all 197 had virtually no other musculoskeletal complaints, our examinations worked from the foot upward in sequence: 1) the foot for lost cuboid subluxation; 2) superior tibio-fibular joint for lost joint-play; 3) knee for passive "springy-block"; 4) sacro-iliac for subluxation and torsion; 5) lumbar spine for damaged lumbar discs; 5) thoracic spine for muscular imbalance and T3 syndromes; 6) cervical spine for disc derangements and Barre's posterior cervical syndrome associated with discs at C2-3 and C3-4.

Results Pedal eversion loss; superior tibio-fibular subluxation; blocked knee movement with ?springy" end-feel denoting loose bodies in virtually all patients. Anterior superior iliac (ASIS) and posterior superior iliac spinal (PSIS) imbalances and "Lock Sign" were usually evident. Warm, swollen, tender PSIS areas and upper lumbar spinal levels indicated disc derangement. Compensatory scoliosis with muscular hypertonus over $\mathrm{T} / 6$ was usually present. The usually neglected costo-transverse syndrome was present in $75 \%$ of patients. Cervical disc derangement, at $\mathrm{C} 2-3$, $\mathrm{C} 3-4$, and $\mathrm{C} 7 / \mathrm{T} 1$, with headaches, dizziness, pain and restricted motion on attempted extension evident almost universally.

Conclusion Once we had precisely identified the involved areas, mobilisation of affected areas, avoidance precautions, and occasional recourse to sclerotherapy achieved prompt?often dramatic, cost-effective, symptomatic amelioration of these vexing problems in 192 patients.

\section{REFERENCES}

1 Cyriax J. Textbook of orthopaedic medicine. 8th edn. London: Balliere \& Tindal, 1988

2 Bowen D, Cassidy M. Spine 1981;6(6):620-8

3 Barre J. Paris Med. 1925;15:266

4 Maigne R. Mid-orthopedic medicine. Illinois: Charles Thomas, 1992

\section{AB0196 THE FREQUENCY OF POPLITEAL CYST IN RA PATIENTS - ULTRASONOGRAPHIC ASSESSMENT}

MR Kowalski. Rheumatology, Medical University of Wrockaw, Wrockaw, Poland 
Objectives The goal of the study was to assess the frequency of Baker's cyst in rheumatoid arthritis patients in comparison to other groups of patients and healthy persons.

Methods With the real time ultrasonographic device with 7,5 $\mathrm{MHz}$ linear probe we examined knee joints of a group of $60 \mathrm{RA}$ patients; 30 health persons served as a control group; the second control group were 60 perimenopausal women.

Results We found Bakre's cysts in 25 out of 60 RA patients (in 5 cases located in both knees). Among healthy persons there was one person with diagnosed popliteal cyst. In a group of perimenopausal women there were two patients with the cyst.

Conclusion This study shows the frequency of Bakre's cyst in RA patients in comparison with other groups of patients. Although the study was conducted on a limited number of patients it can give us an estimated data on Bakre's cyst frequency in RA patients.

\section{AB0197 SONOGRAPHIC ASSESSMENT OF STEROID INJECTION THERAPY}

E Filippucci, A Farina, F Salaffi, C Cervini, W Grassi. Department of Rheumatology, University of Ancona, Jesi, Italy

\subsection{6/annrheumdis-2001.675}

Background Ultrasonography (US) is an excellent technique to look around and inside joints and tendons in patients with chronic arthritis. ${ }^{1}$ Power Doppler imaging combined with conventional grey-scale US could play a role in functional and quantitative assessment of synovitis. ${ }^{2,3}$

Objectives The aim of this study was to assess sonographic changes induced by steroid injection therapy.

Methods Seven joints of 5 patients with active synovitis were studied. A visual analogue scale (VAS) (ranging from 0 to 10) and a tenderness score (ranging from 0 to 10) were assessed as measures of disease activity. Sonographic evaluation was performed with an AU-5 Harmonic, Esaote Biomedica (Genoa, Italy) equipped with a $13-\mathrm{MHz}$ linear transducer. Power Doppler examination was performed just before the ultrasound guided injection with triamcinolone acetonide. Power Doppler settings were standardised with a pulse repetition frequency of $1000 \mathrm{~Hz}$. Colour gain was initially set at a level just below the disappearance of colour noise deep to cortical bone. Joint cavity widening and power Doppler colour signal were scored from 0 to 4 . Ultrasound examination was repeated in the second week after the steroid injection. Statistical evaluation was performed by the Wilcoxon signed-rank test.

Results All the affected joints showed both morphological (joint cavity widening) and functional (colour signal) features of active synovitis. A significant decrease of joint cavity widening $(\mathrm{p}=$ $0.045)$, VAS $(p=0.003)$, and tenderness score $(p=0.0024)$ was detected. Power Doppler score did not show a significant change $(\mathrm{p}=0.091)$. However, a clearly evident decrease of colour signal was observed in 3 out of 5 patients.

Conclusion Our study confirms the clinical efficacy of steroid injection therapy. Grey-scale US seems to be able to carefully detect morphological changes induced by steroid injection therapy. Although this preliminary report shows that power Doppler score is not significantly changed after steroid injection, we believe that further studies on a larger group of patients are needed to better assess the role of power Doppler US in disease activity monitoring.

\section{REFERENCES}

1 Gibbon WW, Wakefield RJ. Ultrasound in inflammatory disease. Radiol Clin North Am. 1999;37:633-51

2 Hau M, Schultz H, Tony HP, Keberle M, Jahns R, Haerten R, Jenett M. Evaluation of pannus and vascularization of the metacarpophalangeal and proximal interphalangeal joints in rheumatoid arthritis by high-resolution ultrasound (multidimensional linear array). Arthritis Rheum. 1999;42:2303-8

3 Newman JS, Laing TJ, McCarthy CJ, Adler RS. Power Doppler sonography of synovitis: assessment of therapeutic response-preliminary observations. Radiology 1996;198:582-4

\section{AB0198 SYNOVIAL FLUID ANALYSIS ? A RETROSPECTIVE REVISION}

PL Nero, MM Costa, JB Pimentao, J Branco. Rheumatology, Egas Moniz, Lisbon, Portugal

\subsection{6/annrheumdis-2001.676}

Background Synovial fluid analysis is an extension of the physical examination. In patients with crystal associated arthritis a few drops of synovial fluid often makes a definite diagnosis.

Objectives The authors reviewed of synovial fluid analysis results of their Department.

Methods A revision is made of all the synovial fluids seen under microscopic ordinary light, polarised light and with alizarin red stain, between the 1st. of September 1999 and the 31th of December 2000. The fluids were taken from in and outpatients of the Rheumatology Department of the Egas Moniz Hospital in Lisbon, who had a formal indication for arthrocentesis.

Results A total of 114 synovial fluids were studied, 76 (66.7\%) women and $38(33.3 \%)$ men. The mean age was $60.8 \pm 16.79$ years. The synovial fluid was intra-articular in $76.8 \%$ and from adjacent bursal tissues in $23.2 \%$. The mean time between arthrocentesis and microscopic analysis of the fluid was $4.72 \pm 7.13$ days. The fluid had mechanical properties in $32.5 \%$ of the cases; different types of crystals in $27.2 \%$; inflammatory properties in $23.7 \%$; $1.75 \%$ were traumatic: $0.88 \%$ were infectious; $14 \%$ were not classified. Half the patients didn't have a previous diagnosis and the synovial fluid microscopic analysis made a definite diagnosis in $40.4 \%$ of these patients: $38.6 \%$ of crystal associated arthritis and $1.85 \%$ of septic arthritis.

Conclusion The synovial fluid microscopic analysis made a definite diagnosis in $40,4 \%$ in patients without a previous one, confirming it is a useful tool in clinical practice.

\section{AB0199 ANTI-TNF MONOCLONAL ANTIBODY USED FOR SCINTIGRAPHIC VISUALISATION OF SYNOVITIS IN RA}

P Barrera, WJ Oyen, OC Boerman, LB Van de Putte, FH Corstens, PL Van Riel. Rheumatology, University Medical Center Nijmegen, Nijmegen, The Netherlands

\subsection{6/annrheumdis-2001.677}

Background Tumour necrosis factor $\alpha(\mathrm{TNF} \alpha)$ is a crucial proinflammatory cytokine present in inflamed joints of rheumatoid arthritis (RA) patients. Therapies using anti-TNF $\alpha$ monoclonal antibodies (MAb) have proven their efficacy in RA and other chronic inflammatory diseases. Such antibodies could also be used to detect inflammation.

Objectives To determine the potential of a ${ }^{99} \mathrm{Tc}$-labelled antiTNF $\alpha$ MAb to image synovitis in patients with RA.

Methods Eight consenting patients with active RA, defined as a disease activity score (DAS) $>3.2$ were enrolled in the study. No previous anti-TNF $\alpha$ therapy was allowed. NSAIDs and lowdose steroids were kept stable during the study. Patients received 